\title{
Bipolar hypershell models of the extended hot interstellar medium in spiral galaxies
}

\author{
Y. Sofue ${ }^{1}$ and A. Vogler ${ }^{2}$ \\ 1 Institute of Astronomy, University of Tokyo, Mitaka, Tokyo 181-0015, Japan \\ 2 CEA/Saclay, DAPNIA, Service d'Astrophysique, L'Orme des Merisiers, Bât. 709, 91191 Gif-sur-Yvette, France
}

Received 23 August 2000 / Accepted 15 January 2001

\begin{abstract}
We simulated the million degree interstellar medium and its soft X-ray images in the disk and halo of spiral galaxies using the bipolar hypershell (BHS) model. In this model dumbbell- or hourglass-shaped expanding shells of several kpc radii are produced by a sudden energy release in the central region. We then applied our model to a mini-sample of starburst galaxies seen under different inclinations, namely the nearly edge-on galaxies NGC 253, NGC 3079 and M 82, the highly inclined galaxies NGC 4258 and NGC 1808 as well as the nearly face-on galaxy M 83. For all galaxies, our results reproduce the X-ray characteristics observed in the $0.1-2.4 \mathrm{keV}$ ROSAT energy band: the bipolar hypershell morphology, the spectral energy distribution of the diffuse disk and halo emission as well as absorption gaps in the diffuse X-ray emission caused by a shadowing of soft X-rays due to cold intervening gas in the disks of the galaxies. In general, the required total energy for the starburst is estimated to be of the order of $10^{55} \mathrm{ergs}$, corresponding to the overall kinetic energy generated in $\sim 10^{4}$ type-II supernova explosions. The expansion velocity of the shells is estimated to be $\sim 200 \mathrm{~km} \mathrm{~s}^{-1}$, which is necessary to heat the gas to $\sim 0.2 \mathrm{keV}(2.3$ million $\mathrm{K})$, and the age to be of the order of $310^{7}$ years. In the case of the very nearby, nearly edge-on galaxy NGC 253 all characteristics of the BHS model can be studied with high spatial resolution. Using the property that the shell morphology is sensitive to the ambient density distribution, we propose using soft X-ray data to probe the gas distributions in the disk, halo and intergalactic space in general. The application of our model to images at higher spatial and spectral resolution, as provided by Chandra and XMM, will help us to further disentangle the ISM density distributions and will lead to a better understanding of the disk halo interface.
\end{abstract}

Key words. galaxies: general - galaxies: ISM - galaxies: starburst - galaxies: spiral - galaxies: structure - X-rays: galaxies

\section{Introduction}

A bipolar-hypershell (BHS) model has been proposed to explain the double-horn features observed in the radio-continuum halo of the starburst galaxy NGC 253 (Sofue 1984). Recent soft-X-ray observations of NGC 253 with ROSAT revealed extended outer-halo emission with double-horn morphology, and is interpreted as being a hypershell driven by a central starburst (Vogler \& Pietsch 1999a; Pietsch et al. 2000). Many spiral galaxies exhibit similar galactic-scale outflows in the form of dumbbellshaped hypershells, bipolar cylinders, or galactic-scale jets. Examples of prototypes of such galaxies are M 82 (NGC 3034), NGC 1808 and NGC 3079 (see next section for references). Starburst-origin models for extended halo features in galaxies were proposed by several authors

Send offprint requests to: Y. Sofue, e-mail: sofue@ioa.s.u-tokyo.ac.jp
(Heckman et al. 1993; Tomisaka \& Ikeuchi 1987; Suchkov et al. 1994, 1996).

The Milky Way also exhibits bipolar hypershells extending above and below the galactic plane in soft X-rays (Snowden et al. 1997) and radio continuum (e.g. Haslam et al. 1982), which are also interpreted as an effect of a giant $\Omega$-shaped shock front originating from a starburst some $10^{7}$ years ago in the Galactic Center (Sofue 1977, 1984, 1994, 2000). These hypershells and flows, including that in the Milky Way, require a similar amount of total energy of the order of $\sim 10^{55}$ ergs corresponding to the energy release from $10^{4}$ to $10^{5}$ type II supernovae. This fact suggests that they may have a common property and origin, and can be modeled by a unified scheme of energy release in the galactic center and its propagation into the halo and intergalactic space.

In this paper, we simulate the extended soft X-ray halo components in various galaxies based on the bipolar hypershell (BHS) model developed for the Milky Way halo 
(Sofue 1977, 1984, 1994, 2000). We further propose to use the simulation to date and measure the explosive events at the nuclei, such as their starburst history. The simulation can also be used to obtain information about the galactic-halo-intergalactic interface in disk galaxies. The X-ray absorption morphology near the galactic plane can be used to probe the interstellar gas distribution in the galactic disk.

\section{Soft X-ray hypershells in nearby spiral galaxies}

\subsection{NGC 253}

ROSAT images of the nearby $(d=2.6 \mathrm{kpc}$, Puche \& Carignan 1988) starburst galaxy NGC 253 revealed diffuse $\mathrm{X}$-ray emission from the nuclear region, the disk and the halo hemispheres (Pietsch et al. 2000). The emission measures of the three components are nearly identical, and the integral diffuse emission $\left(L_{\mathrm{x}}=410^{39} \mathrm{erg} \mathrm{s}^{-1}\right)$ accounts for $\sim 80 \%$ of the total X-ray luminosity from NGC 253 . The diffuse bulge emission is due to an extended, highly absorbed source with an extent of $250 \mathrm{kpc}$, and a hollowcone shaped "X-ray plume" with an extent of $\sim 700 \mathrm{pc}$ along the SE minor axis. The X-rays in the bulge region are thought to trace the interaction between a galactic super-wind driven by the starburst nucleus and dense interstellar medium in the disk.

In the disk, the highly absorbed diffuse emission follows the spiral arm structure. It most probably reflects a superposition of diffuse X-ray emission from hot interstellar and discrete sources, such as emission from binaries, SN-remnants or HII regions. Two major components of the diffuse halo emission can be separated: "coronal emission" arising from hot interstellar medium close to the disk (scale height $\sim 1 \mathrm{kpc}$ ) and emission from the "outer halo". NGC 253 is not completely oriented edge-on, and the NW side of the galaxy is closer to us. Due to this viewing geometry and absorption of the soft X-rays by the disk of NGC 253, the coronal emission can only be detected from the near (SE) side of the galaxy, while emission from the outer halo component is partly shielded by the HI disk of NGC 253 to the NW. Following Pietsch et al. (2000), the X-ray corona might be fueled by hot gas from galactic fountains within the disk, while the outer halo emission is attributed to a superwind emanating from the starburst nucleus. The latter assumption can also explain the "hourglass-" or "dumbbell-shaped" morphology of the outer X-ray halo. Pietsch \& Vogler (2000) estimate temperature of the outer halo component at $0.2 \mathrm{keV}$ (2.3 million $\mathrm{K})$, corresponding to an equivalent shock-heating velocity of $200 \mathrm{~km} \mathrm{~s}^{-1}$.

\subsection{NGC 1808}

NGC 1808 is a galaxy with large-scale cylindrical dusty jets emanating from the nucleus at high velocities, and is supposed to be a mildly tilted M 82 type galaxy (Véron-Cetty \& Véron 1985; Phillips 1993). Soft X-ray imaging observations with the ROSAT PSPC and HRI revealed an active X-ray nucleus and extended emission in the disk region (Junkes et al. 1995; Dahlem et al. 1994), as well as a very extended X-ray emission in the halo (Iwaki et al. 1996). The total soft X-ray luminosity is of the order of $310^{40} \mathrm{ergs} \mathrm{s}^{-1}$. Iwaki et al. (1996) obtained wide area ASCA images in the $0.5-2 \mathrm{keV}$ band, and found that the extended component is highly elongated in the direction of the dusty jets on both sides of the galactic plane. Furthermore, the X-ray halo appears to be bifurcated into two ridges, suggesting that it comprises double-horn feature or a partial shell structure.

\subsection{NGC 3079}

The nearly edge-on galaxy NGC 3079 is known to host an active nucleus classified as a LINER or Seyfert II nucleus, and giant radio lobes and $\mathrm{H} \alpha$ superbubbles emanate along the minor axis (Irwin \& Seaquist 1991; Cecil et al. 1995). ROSAT observations (Pietsch et al. 1998) detected an integral $0.1-2.4 \mathrm{keV}$ luminosity of $L_{\mathrm{x}}=310^{40} \mathrm{erg} \mathrm{s}^{-1}$, which can be resolved into three components. One component consists of extended emission from the central region $\left(L_{\mathrm{x}}=110^{40} \mathrm{erg} \mathrm{s}^{-1}\right)$ originating from the region of the central superbubble. While most of this emission is thought to represent hot gas in the superbubble, one cannot exclude additional contributions of $\leq 30 \%$ from the active nucleus. The second main emission component arises from the disk of the galaxy $\left(L_{\mathrm{x}}=710^{39} \mathrm{erg} \mathrm{s}^{-1}\right)$ and can be partly resolved into point-like sources. Each of the three detected point sources has $L_{\mathrm{x}} \sim 610^{38} \mathrm{erg} \mathrm{s}^{-1}$. Finally, emission from the halo $\left(L_{\mathrm{x}}=610^{39} \mathrm{erg} \mathrm{s}^{-1}\right)$ is detected. As in the case of NGC 253, the hot gas does not fill the halo hemispheres uniformly, but an hourglass-shaped structure of the halo emission is reported. A mean temperature for the halo gas was estimated to be $3.510^{6} \mathrm{~K}$. Interestingly, the lowest contours of the extended emission show a saddle shape, with a neck at the galactic plane, suggestive of bipolar double-horns.

\subsection{NGC 4258}

NGC 4258's anomalous arms could also be due to out-ofplane partial shells (van Albada \& van der Hulst 1982; Vogler \& Pietsch 1999b). Diffuse X-ray emission of the galaxy was detected with $L_{\mathrm{x}} \sim 210^{40} \mathrm{erg} \mathrm{s}^{-1}$ and fills $\sim 40 \%$ of the $D_{25}$ diameter of NGC 4258 . At least one half of the diffuse emission is caused by the anomalous spiral arms of this galaxy, and the X-ray emission morphology closely follows the pattern detected in the radio wavelengths.

In the literature, those arms are discussed to be (a) jets confined to the disk of the galaxy and expelled by the active nucleus (e.g., Martin et al. 1989; Hummel et al. 1989; Plante et al. 1991; Cecil et al. 1995), (b) outflows (jets or plumes) from the active nucleus leaving the plane of the galaxy and possibly "raining down" onto the disk again 
(van der Kruit et al. 1972), (c) a hypershell of hot interstellar medium as present in the nuclear region of NGC 253 and expanding from the disk into the halo (Sofue 1984, 2000), or (d) bar shocks heating the interstellar medium due to the high differential velocities between the bar orbit and interstellar medium in the bulge and inner disk of NGC 4258 (e.g., Cox \& Downes 1996).

Remaining diffuse emission not correlated with the anomalous arms might originate from the disk and the halo of the galaxy. For a possible halo component, the temperature was estimated to be $210^{6} \mathrm{~K}$. This temperature is lower by a factor of 2 than the temperature of the hot gas along the anomalous spiral arms $\left(T \geq 410^{6} \mathrm{~K}\right)$.

In addition to the diffuse emission, 14 point sources (integral $L_{\mathrm{x}} \sim 210^{39} \mathrm{erg} \mathrm{s}^{-1}$ ) were found in the galaxy. For possible contributions of the active nucleus to the X-ray emission, an upper limit of $L_{\mathrm{x}} \sim 110^{38} \mathrm{erg} \mathrm{s}^{-1}$ was found in the ROSAT $0.1-2.4 \mathrm{keV}$ band.

\section{5. $M 82$}

M 82 is an edge-on starburst galaxy, which ejects a galactic-scale flow through bipolar cylindrical jets (Nakai et al. 1987) to which larger-scale X-ray extended features are associated (Lehnert et al. 1999). Strickland et al. (1997) report the detection of a galactic wind. The $\mathrm{X}$-ray emission was visible out $\geq 6 \mathrm{kpc}$ above and below the plane of the galaxy, and the luminosity of the diffuse $\mathrm{X}$-ray emission outside the nuclear region is $\sim 210^{40}$ erg $\mathrm{s}^{-1}$. From X-ray spectra, the temperature of the gas is found to vary from $0.6 \mathrm{keV}$ near the nucleus to $0.4 \mathrm{keV}$ for distances further away from the plane. The authors compared the X-ray results with several theoretical models, namely Tomisaka \& Ikeuchi (1988), Tomisaka \& Bregman (1993); Suchkov et al. (1994). The model of Suchkov et al. (1994) assumes a two-component cold rotating dense disk, a non-rotating hot tenuous halo, and a starburst history incorporating the milder mass and energy input from stellar winds into the halo before more energetic supernova explosions dominate the scenario. Shocked halo gas then provides the major emission component in the ROSAT band.

A reanalysis of the ROSAT data (Lehnert et al. 1999) and new $\mathrm{H} \alpha$ images showed the existence of a $4 \mathrm{kpc} \times 1 \mathrm{kpc}$ region of spatially coincident $\mathrm{X}$-ray and $\mathrm{H} \alpha$ emission about $10 \mathrm{kpc}$ north of the nucleus. The luminosity in both wavebands accounts for roughly $1 \%$ of the total M 82 luminosities in both bands. The authors attribute the $\mathrm{H} \alpha$ emission to ionizing radiation from the starburst that propagates into the halo, and the X-ray emission to a shock-heated massive ionized cloud in the halo, which is encountered by the superwind. With this scenario, they follow theoretical predictions of Suchkov et al. (1996).

\subsection{The Milky Way}

Using ROSAT All Sky Survey (RASS) data, presented in several energy bands from 0.1 to $2.4 \mathrm{keV}$ in Snowden et al. (1997), we showed that the North Polar Spur (NPS) and its western and southern counter-spurs trace a giant dumbbell-shape with a neck at the galactic plane (Sofue 2000). These features are interpreted as effects of a shock front originating from a starburst 15 million years ago with a total released energy of the order of $\sim 10^{56}$ ergs or $10^{5}$ type II supernovae. We simulated all-sky distributions of radio continuum and soft X-ray intensities based on the bipolar hypershell galactic center starburst model. The simulations nicely reproduce the radio NPS and related spurs, as well as radio spurs in the tangential directions of spiral arms. Simulated X-ray maps in $0.25,0.75$ and $1.5 \mathrm{keV}$ bands reproduce the ROSAT X-ray NPS, its western and southern counter-spurs, and the absorption layer along the galactic plane. We further proposed to use the ROSAT all-sky maps to probe the physics of gas in the halo-intergalactic interface of the Milky Way, and to directly date and measure the energy of a recent Galactic Center starburst. The present paper is a series of the BHS modeling of extended halo X-rays in galaxies using the same numerical code.

\section{Bipolar hypershell starburst model}

We adopt the same numerical methods to trace the shock envelope and X-ray intensity distributions as we used to model the radio spurs and BHS in the Milky Way, which is described in detail in Sofue (2000). We briefly describe the methods below.

\subsection{Adiabatic-shock envelope method}

The propagation of a shock wave through the galactic halo induced by a point energy injection at the center can be calculated by applying the shock-envelope-tracing method of Sakashita (1971) and Möllenhoff (1976), who extended the Laumbach \& Probstein (1969) method for tracing the evolution of a shock front to a case of axi-symmetric distributions of ambient gas. The flow field is assumed to be locally radial, and the gas is adiabatic, and, therefore, the heat transfer by radiation and counter-pressure are neglected. The density contrast between the shock front and ambient gas is given by $(\gamma+1) /(\gamma-1)=4$ for $\gamma=5 / 3$, where $\gamma$ is the adiabatic exponent of the gas.

The equation of motion of the shock wave is given as follows (Möllenhoff 1976):

$E_{0}=\int_{0}^{R} \frac{P}{\gamma-1} 4 \pi r^{2} \mathrm{~d} r+\int_{0}^{R} \frac{1}{2}\left(\frac{\partial r}{\partial t}\right)^{2} \rho_{0} 4 \pi r_{0}^{2} \mathrm{~d} r_{0}$.

Here, $E_{0}$ is the total energy of the explosive event, $P$ is the internal pressure, $\gamma$ is the adiabatic exponent, $\rho_{0}$ is the unperturbed ambient gas density, and $r$ is the radius from the explosion center, with suffix 0 denoting the quantities of the unperturbed ambient gas, and $R$ is the radius of 
Table 1. Parameters for shock envelope solutions

\begin{tabular}{cccccccc}
\hline Model & $\begin{array}{c}\text { Energy } \\
\mathrm{ergs}\end{array}$ & $\begin{array}{c}\rho_{1} \\
\mathrm{H} \mathrm{cm}^{-3}\end{array}$ & $\begin{array}{c}h_{1} \\
\mathrm{kpc}\end{array}$ & Disk $z$ type & $\begin{array}{c}\rho_{2} \\
\mathrm{H} \mathrm{cm}^{-3}\end{array}$ & $\begin{array}{c}h_{2} \\
\mathrm{kpc}\end{array}$ & $\begin{array}{c}\rho_{3} \\
\mathrm{H} \mathrm{cm}^{-3}\end{array}$ \\
\hline S1 & $0.410^{55}$ & 1 & 0.1 & exponential & 0.01 & 1 & $10^{-5}$ \\
S2 & $"$ & 1 & 0.2 & exponential & 0.01 & 1 & $"$ \\
S3 & $"$ & 1 & 0.1 & Gaussian & 0.01 & 1 & $"$ \\
S4 & $"$ & 1 & 0.2 & Gaussian & 0.01 & 1 & $"$ \\
S5 & $"$ & 1 & 0.1 & power law & 0.01 & 1 & $"$ \\
S6 & $1.610^{55}$ & 0.1 & 2 & $\exp \left[-\left(z / h_{1}\right)^{4}\right]$ & 0.0 & - & $"$ \\
\hline
\end{tabular}

The shock envelope (radius and velocity) obeys a scaling law: for equal ratios $E / \rho_{i}$, one obtains the same envelope, independent of the individual $E, \rho_{i}$ values.

the shock front. This equation then leads to an equation of the shock radius, as described in Sofue (2000).

The unperturbed density distribution of gas in a galaxy is assumed to comprise a stratified disk, a halo with an exponentially decreasing density, and intergalactic gas with a uniform density. The differential distribution is approximated by the following expression.

$\left.\rho_{0}=\rho_{1} \exp \left(-\left(z / z_{1}\right)^{\eta}\right)\right)+\rho_{2} \exp \left(-z / z_{2}\right)+\rho_{3}$.

Here, suffices 1, 2 and 3 denote quantities for the disk, halo and intergalactic gas, respectively, $\rho$ is the density, $z$ is the height from the galactic plane, $z_{i}$ is the scale thickness of the disk and halo. The power index $\eta$ represents the tightness of the disk component toward the galactic plane, and is taken either 1 (exponential) or 2 (Gaussian). The third component, representing the intergalactic gas density, is taken to be constant at $\rho_{3}=10^{-5} \mathrm{H} \mathrm{cm}^{-3}$, while the other parameters are set in various possible combinations as listed in Table 1. We also calculate a case in which the halo density decreases with a power law. We further calculate another case with uniform halo density which decreases suddenly at a certain height facing the intergalactic gas, which represents a case for halo gas confined by the intergalactic gas pressure.

Solutions of the above equations are presented in Fig. 1 for various parameter sets given in Table 1 . The total energy input is taken to be $E_{0}=0.410^{55}$ ergs for S1 to S5, and $1.610^{55}$ ergs for S6, and the shock envelopes are drawn every 10 million years. The shock velocity can be estimated by dividing the radial distance between succeeding envelopes by $10^{7}$ years.

Models S1 and S2: Exponential disk with scale height 0.1 and $0.2 \mathrm{kpc}$, respectively, exponential halo with scale height $1 \mathrm{kpc}$, and intergalactic gas of uniform density.

Models S3 and S4: Gaussian disk with half thickness 0.1 and $0.2 \mathrm{kpc}$, respectively, exponential disk of scale height $1 \mathrm{kpc}$, and intergalactic gas of uniform density.

Model S5: Power-law density disk, power-law density halo, and intergalactic gas of uniform density.

Model S6: Disk of $2 \mathrm{kpc}$ thickness with sudden decrease of density, facing the intergalactic low-density uniform gas. This model represents a case where the halo-to-disk gas is not gravitationally stratified (e.g., as exponentially), but is confined by intergalactic gas pressure, so that the internal pressure and density in the halo are more uniform.

\subsection{X-ray emission and absorption}

The X-ray intensity distribution for a model galaxy having a hypershell is calculated as below. The centers of the shells are assumed to be at $z= \pm 6 \mathrm{kpc}$ on the rotation axis, and the radii 6 and $9 \mathrm{kpc}$ in the radial and vertical directions, respectively. The volume emissivity is calculated from the density contrast of the shocked gas, which is assumed to be proportional to $\rho / \rho_{0}=(\gamma+1) /(\gamma-1)$, where $\rho$ and $\rho_{0}$ are gas densities in the shocked shell and unperturbed halo gas, respectively. In the present simulation, the profile of emissivity perpendicular to the shell surface is simply represented by an exponentially decreasing function behind the shock front toward the center with a scale thickness of $500 \mathrm{pc}$. The emissivity also decreases with the height from the galactic plane with a scale height of $3 \mathrm{kpc}$, corresponding to the exponentially decreasing gas density in the halo.

The X-ray emission from a hypershell is assumed to be thermal free-free radiation, whose emissivity is given by

$\epsilon=n_{\mathrm{e}}^{2} \Lambda \propto \rho^{2} T^{1 / 2}$

Here, $\Lambda$ is the cooling function of the gas. Since the temperature is as high as $T \sim 10^{7} \mathrm{~K}$ in our simulation, the radiation is almost totally free-free, and the contribution by recombination lines from hydrogen, helium, and metals, which are significant at $T \sim 10^{5} \mathrm{~K}$, can be neglected. A typical emission measure along the hypershell ridge is $n_{\mathrm{e}}^{2} L \sim 10^{-2} \mathrm{~cm}^{-6}$ pc for a tangential pass in the shell of about 2 kpc. For simplicity, we assume that the temperature of X-ray emitting gas is constant in the shocked shell. In addition to the hypershells, we assume a galactic disk X-ray component of scale height of $500 \mathrm{pc}$, and a bulge component of scale radius $1 \mathrm{kpc}$. X-ray emissions from the ambient (unperturbed) halo gas is neglected, because the density would not be sufficiently high to contribute a significant $0.1-2.4 \mathrm{keV}$ X-ray emission measure.

The X-ray intensity, $I$, is calculated by

$\mathrm{d} I=\epsilon_{\mathrm{X}} \mathrm{d} s-\kappa I \mathrm{~d} s$, 

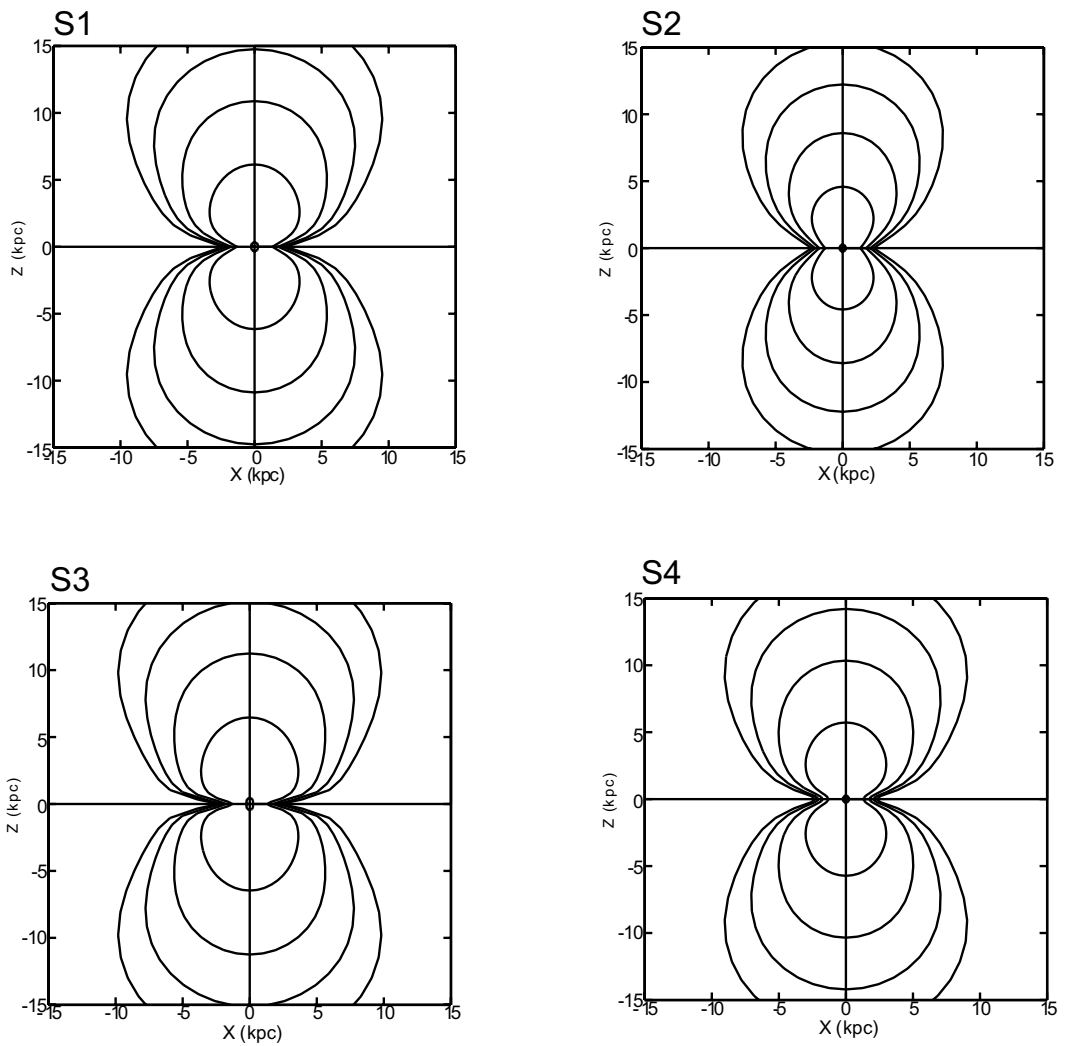

S5

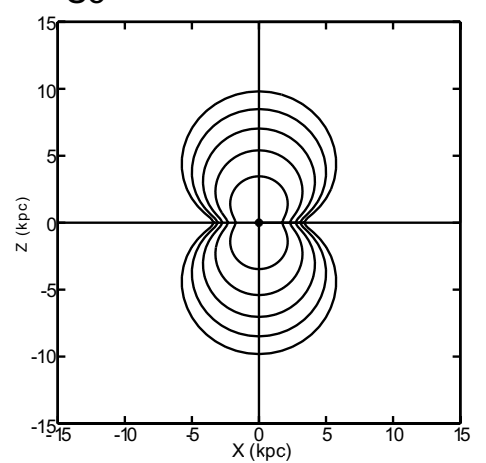

S6

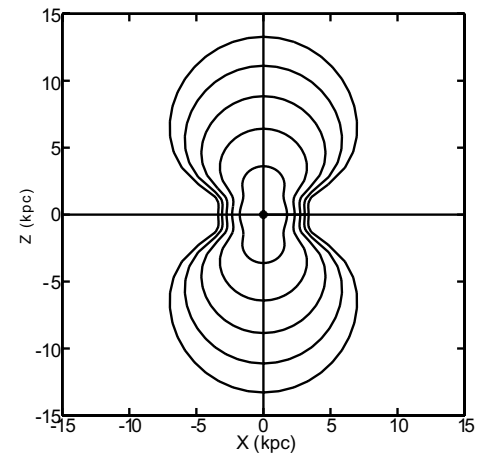

Fig. 1. Calculated shock front in the galactic halo at every $2.510^{7}$ yrs after an explosion and/or a starburst at the nucleus with a total energy of $0.410^{55}$ erg for various parameters as models S1 to S6 in Table 1

where the first term in the right-hand side is the emission measure with $\epsilon_{\mathrm{X}}$ being the X-ray emissivity, and the second term represents the absorption rate with $\kappa$ being absorption coefficient, and $s$ is the distance along the lineof-sight.

The interstellar extinction of soft X-rays occurs due to the photoelectric absorption by metals, and has been calculated for the solar metal abundance by Morrison \& McCammon (1983). The absorption coefficient $\kappa$ is, then, represented by

$\kappa=\left(n_{\mathrm{H}} / N_{\mathrm{H}}^{0}\right)\left(E^{\mathrm{X}} / E_{0}^{\mathrm{X}}\right)^{-2.5}$ where $n_{\mathrm{H}}$ is the number density of hydrogen atoms, and $N_{\mathrm{H}}^{0}$ is the $e$-folding column density of interstellar neutral hydrogen at the photon energy $E_{0}^{\mathrm{X}}$ (Ryter 1996; Sofue 2000). At $E_{0}^{\mathrm{X}}=0.75 \mathrm{keV}$, the $e$-folding column density for $E_{0}^{\mathrm{X}}=0.75 \mathrm{keV}$ is given as $N_{\mathrm{H}}^{0}=310^{21} \mathrm{H} \mathrm{cm}^{-2}$ (Snowden et al. 1997). Since the ROSAT energy bands are rather broad, covering higher and lower energies around the representative energies, we adopt two representative cross sections at 0.75 and $1.5 \mathrm{keV}$ bands, which are 1 and 0.1 times the value at $0.75 \mathrm{keV}$, respectively.

The absorbing disk gas is assumed to be condensed in logarithmic spiral arms of pitch angle $6^{\circ}$ and the width is taken to be $1 / 5$ of the arm separation, as in the same 
way assumed for the Milky Way. The peak hydrogen density in an arm at a distance of $8 \mathrm{kpc}$ from the center is taken to be $5 \mathrm{H} \mathrm{cm}^{-3}$. The hydrogen density distribution is expressed by

$n_{\mathrm{H}}=\alpha n_{0} \exp \left(-r / r_{\mathrm{H}}-z / z_{\mathrm{H}}\right) \cos ^{k}\left(\theta-\eta \log r / r_{0}\right)$.

Here, $\theta$ and $\eta$ are the azimuthal angle and the pitch angle of the arms.

\section{Results of simulations}

\subsection{NGC 253}

The expansion velocity of the outer halo is estimated to be $200 \mathrm{~km} \mathrm{~s}^{-1}$, which is required for shock heating of the gas up to $0.2 \mathrm{keV}\left(2.310^{6} \mathrm{~K}\right)$ (Pietsch et al. 2000). Taking this expansion velocity for the BHS at $5 \mathrm{kpc}$ height from the galactic plane in the ROSAT $0.75 \mathrm{keV}$ contour map, we find that the observation is well fitted by a shock front at $\sim 310^{7}$ yrs for an explosion energy of $\sim 410^{54} \mathrm{ergs}$ as in Table 1.

Among the models, however, a better fit is obtained by Models S2 and S6 than by the other models, which indicates that the decrease of halo gas density in the direction perpendicular to the disk is not as steep as in the other models. In Fig. 2 we show the shock front for Models S2 and S6 superposed on the $0.75 \mathrm{keV}$ ROSAT map of NGC 253 in contours overlaid on a $B$-band optical photograph from DSS. The ROSAT result shows that the hypershells tend to break and become round at high altitudes, when the shock front reaches a few kpc from the galactic plane, mimicking a dumbbell shape. Hence, Model S6 appears to be preferable to $\mathrm{S} 2$, for which the BHS is more like an "hourglass". Model S6 represents a case in which the gas density is uniform within the disk and halo up to a certain height (e.g. $2 \mathrm{kpc}$ ), where the halo faces a uniform intergalactic gas of lower density. Such density distribution will be possible if the halo gas is confined by the intergalactic gas pressure, but not stratified by a hydrostatic equilibrium under the disk gravity.

Although Model S6 appears better, overall reproduction of the observations is obtained by both models. For simplicity, we hereafter use Model S2 in which the shape of the shell can be well approximated by a part of an ellipsoidal sphere. This allows us a prediction for the X-ray emissivity and density distributions. Figure 3 shows the calculated intensity distributions for NGC 253 for two different opacity $(\kappa)$ values, which correspond to (a) a case of $0.75 \mathrm{keV}$ band, and (b) $1.5 \mathrm{keV}$ band. We also show the ROSAT X-ray images in the right panels: right-top for $0.75 \mathrm{keV}$ in gray scale, and the right-middle and rightbottom are for 0.75 and $1.5 \mathrm{keV}$, respectively, overlaid on an optical photograph. As seen in the figures, the BHS simulation reproduces the following observed characteristics: (1) double-horn morphology of the diffuse emission features in the $0.75 \mathrm{keV}$ band emerging from the disk, (2) strong absorption along the galactic plane, and (3) energy
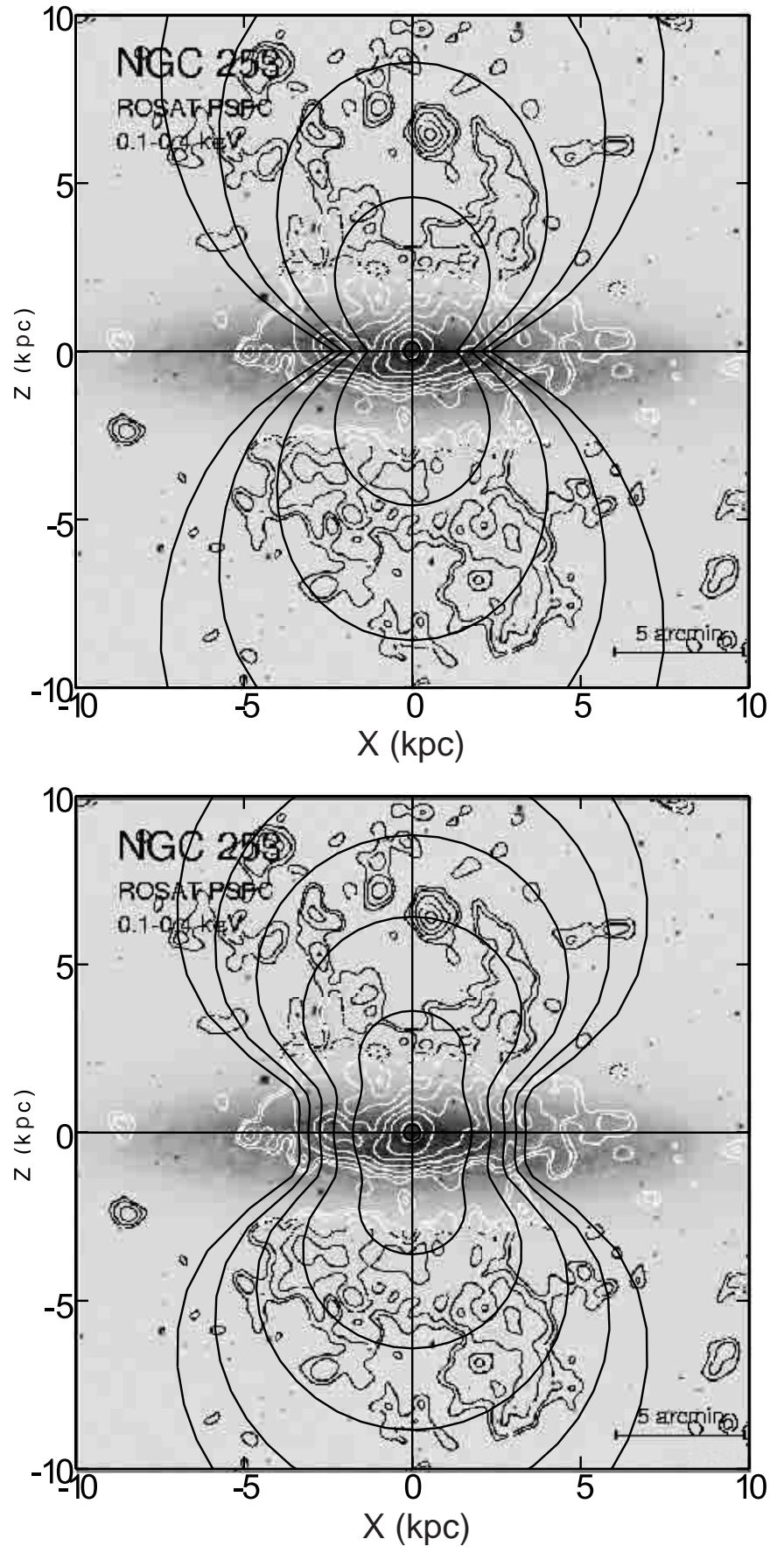

Fig. 2. Calculated shock fronts for Model S2 and S6 overlaid on the ROSAT $0.75 \mathrm{keV}$ contours and optical photograph of NGC 253 from Pietsch et al. (2000)

dependence of the intensity distribution, namely, harder emission near the disk.

The Combination of three NGC 253 images in independent energy bands centered at $0.25,0.75$ and $1.5 \mathrm{keV}$ enabled the construction of a true color image (Pietsch et al. 2000). For this purpose, the different subbands were associated with the colors red, green and blue, for the $0.25,0.75$ and $1.5 \mathrm{keV}$ band, respectively. Figure 4 shows the thus obtained colored intensity distribution compared to the observed spectral intensity distribution taken from 

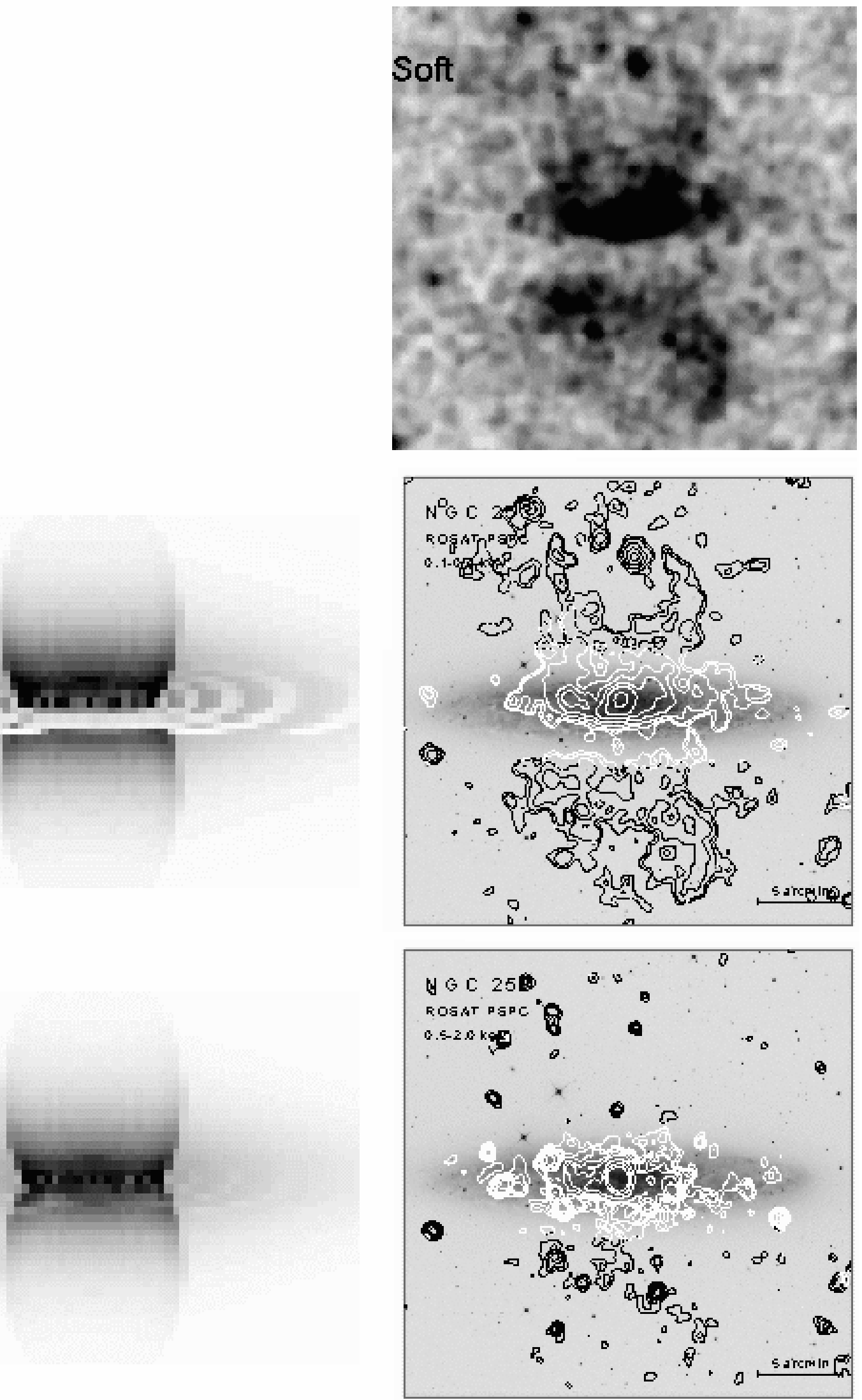

Fig. 3. Simulated intensity distributions for NGC 253 at 0.75 and $1.5 \mathrm{keV}$ (left panel) compared with ROSAT images at 0.75 and $1.5 \mathrm{keV}$ taken from Pietsch et al. (2000) (right panel) 

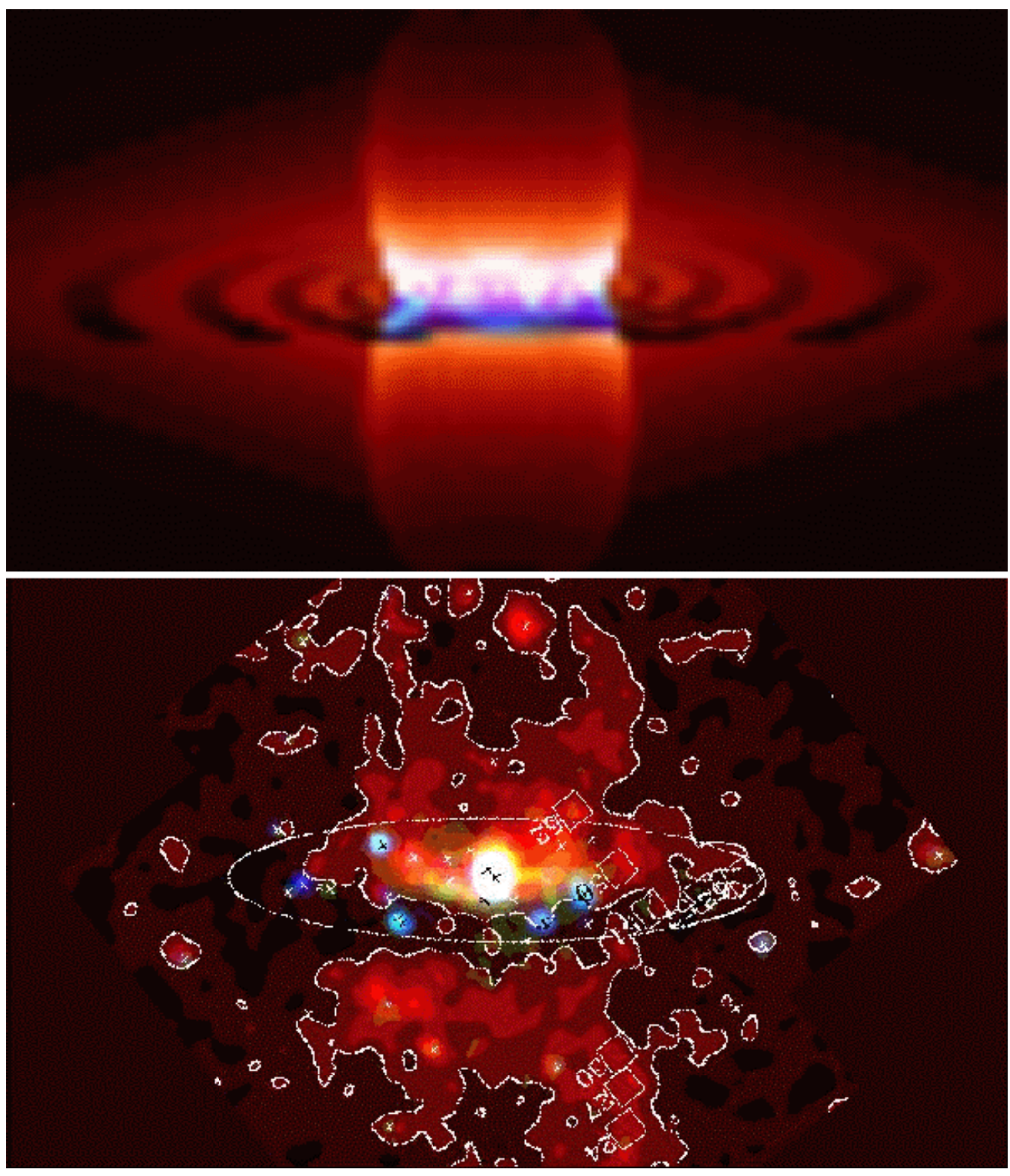

Fig. 4. Addition of 0.25 (R), $0.75(\mathrm{G})$ and 1.5 (B) keV intensities, compared with that of NGC 253 (Pietsch et al. 2000)

Pietsch et al. (2000), where red stands for soft and blue for hard. The present BHS model can nicely reproduce the observed spectrum distribution, such as the harder spectra in the disk and spiral arms due to stronger absorption of softer X-rays.

\subsection{BHS at various inclination: comparison with other galaxies}

We have thus shown that the extended X-ray features in the halo of NGC 253 are well reproduced using the BHS model, which was originally used to model the North Polar Spur (NPS) and associated spurs in the Milky Way halo (Sofue 2000). It is, therefore, interesting to see, whether the BHS model also applies to extended X-ray features observed in other galaxies exhibiting starbursts and outflows. Here, we compare the observed morphology with the outcomes of our theoretical description. We calculated
$0.75 \mathrm{keV}$ intensity distributions for various inclination angles at $0^{\circ}$ (face-on), $30^{\circ}, 45^{\circ}, 60^{\circ}, 80^{\circ}$, and $90^{\circ}$ (edge-on). Figure 5 shows the calculated results, and they are compared in Fig. 6 with observed examples for M $83\left(i=24^{\circ}\right)$, NGC $1808\left(i=58^{\circ}\right)$, NGC $4258\left(i=67^{\circ}\right)$, NGC 253 $\left(i=78^{\circ}\right)$, NGC 3079 (edge-on), M 82 (edge-on) and the Milky Way (edge-on).

$M$ 83: When a galaxy with BHS is observed face-on, the X-ray images exhibit a bright ring feature as in Fig. 5. If the galaxy is slightly inclined such as $i=30^{\circ}$, the BHS ridges in both sides of the galactic plane are superposed to exhibit a box-shaped bright region. Such a boxshaped X-ray distribution appears to be observed indeed in the ROSAT PSPC image of M 83, as shown in Fig. 6 (Strickland et al. 1997), where the arrow points toward the direction of the minor axis as the near side. Although it is difficult to distinguish the halo emission from disk contributions, spiral arms and/or the bar, some emission 

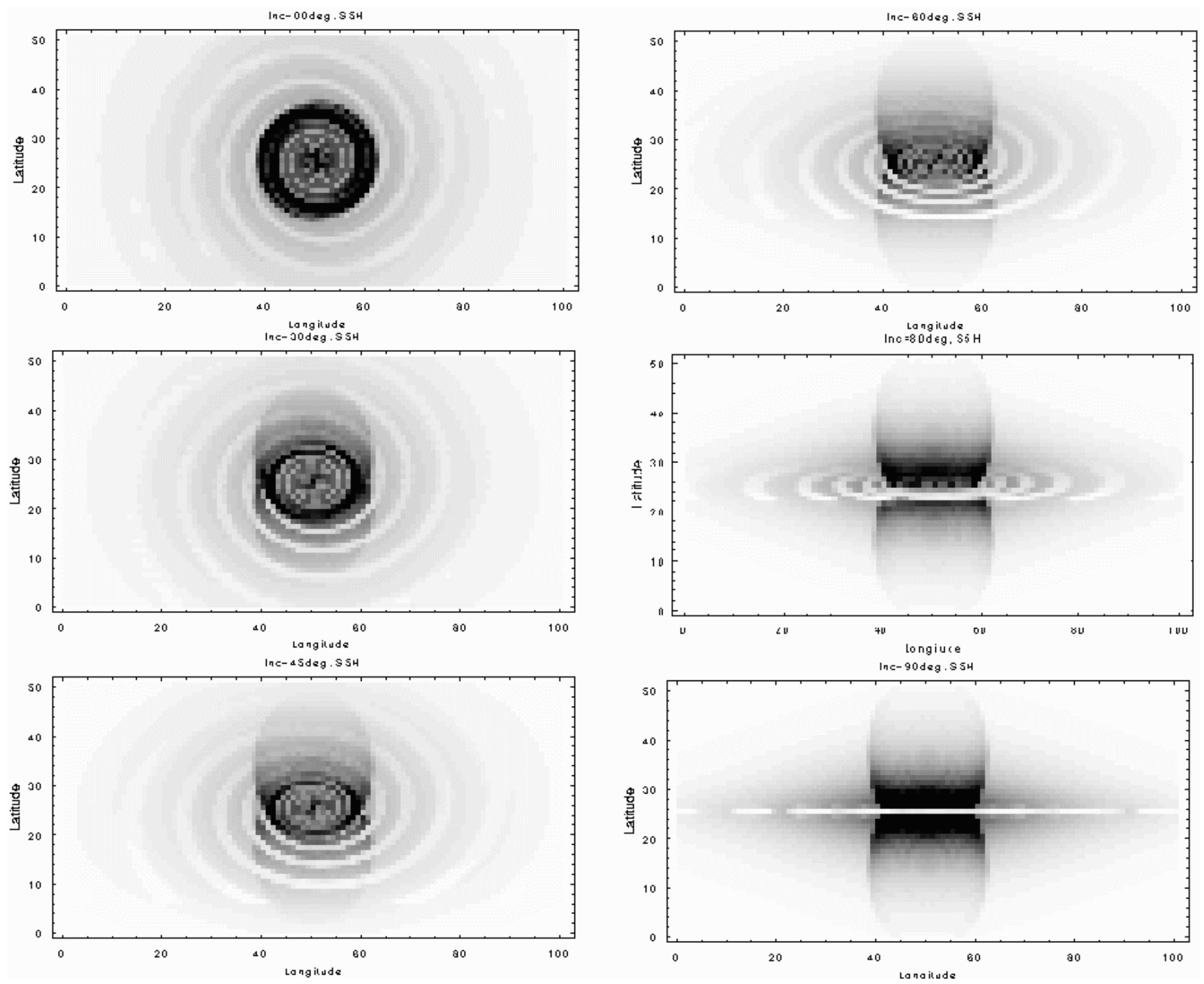

Fig. 5. Simulated $0.75 \mathrm{keV}$ views of a galaxy at various inclination angles

along the boxy edges could be due to overlapped ridges of BHS.

$N G C$ 1808: The extended $0.5-2 \mathrm{keV}$ features bifurcated in the halo of NGC 1808 as observed with ASCA (Iwaki et al. 1996) are also reproduced by our simulations, although the lopsidedness cannot be reproduced well. The arrow in Fig. 6 indicates the direction of the dusty jet along the minor axis in the nearer side.

$N G C$ 4258: This is a galaxy known for its anomalous radio arms, along which $\mathrm{X}$-ray extended emission is observed, as is shown in the 0.1-0.4 keV ROSAT map (Vogler \& Pietsch 1999b). Although the resolution is still too crude, some spur features are mimicked by the BHS model. The origin of the anomalous arms is controversial, as described in Sect. 2, but we comment that the anomalous radio arms (van Albada \& van der Hulst 1982) are nicely fitted by two oppositely extending ridges of the BHS. In particular, their round shapes and curvature of the arms returning to the disk can be explained if they are part of BHS. However, the lobsidedness of the anomalous arms with respect to the rotation axis remains a question in the BHS model, although some lopsidedness is seen in the BHS of the Milky Way (Fig. 6 bottom, constructed from the energy bands presented in Snowden et al. 1997).

$M$ 82: The ROSAT HRI image (Strickland et al. 1997) shows a bipolar extended halo elongated in the direction perpendicular to the disk, and the emission appears to be centrally filled, indicating that the outflow is well collimated, as well simulated by the "jet-like wind" model by Suchkov et al. $(1994,1996)$. We mention, however, that the northern end of the flow at a height of about $2.5 \mathrm{kpc}\left(3^{\prime}\right)$ from the disk appears to be more opened into the intergalactic space, suggesting double-horn ridges or a partial shell.

NGC 3079: The radio-lobe edge-on galaxy NGC 3079 shows a box-shaped X-ray features, suggesting a 

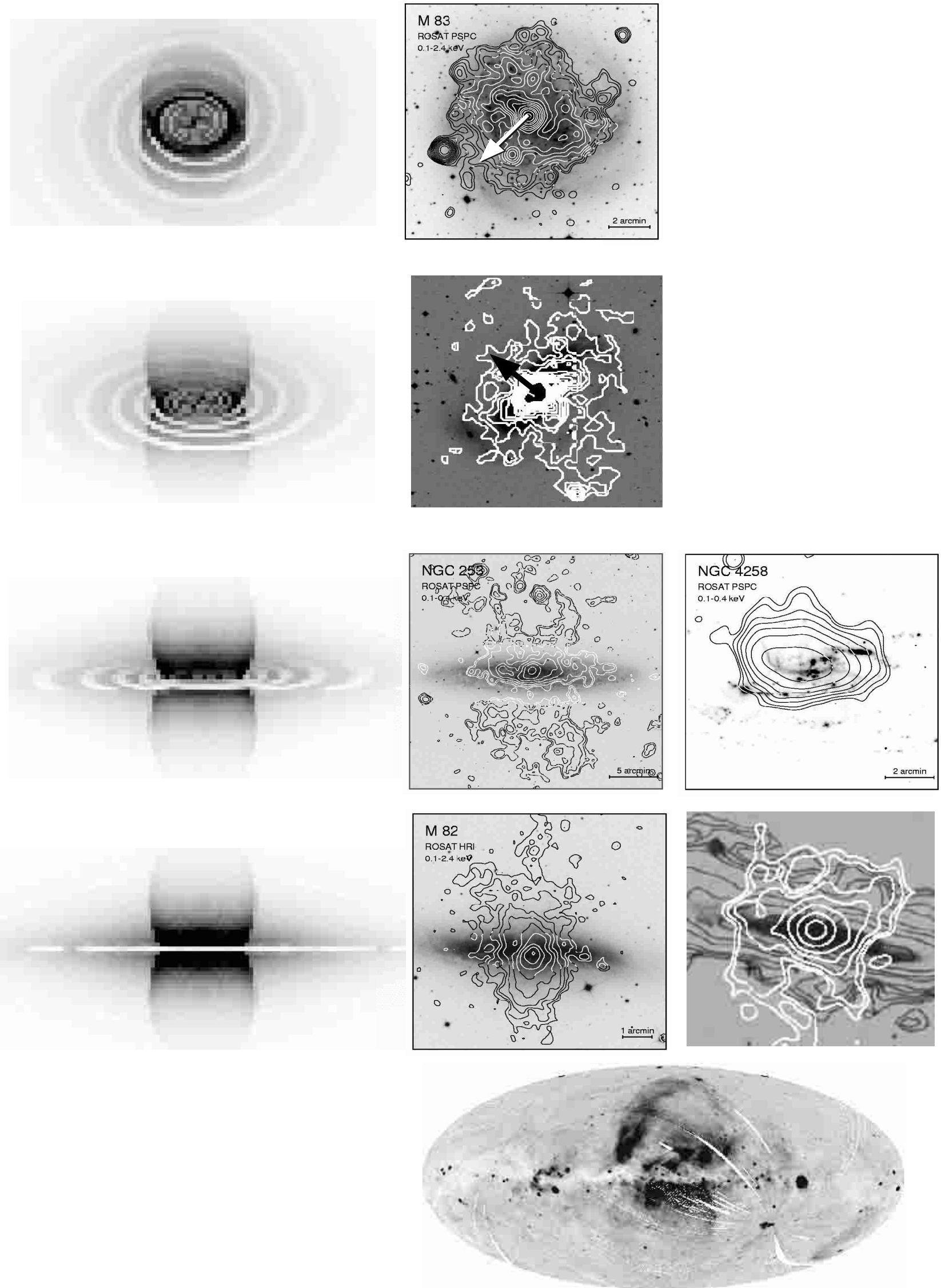

Fig. 6. Comparison of the model with observed X-ray images of several galaxies with various inclinations: from top to bottom, M 83, NGC 1808, NGC 253, NGC 4258, M 82, NGC 3079, and the Milky Way. The arrows in M 83 and NGC 1808 point the directions of the minor axis in the near side. See the text for references 
double-horn morphology, as observed in the ROSAT PSPC image (Pietsch et al. 1998), and is well reproduced by an edge-on BHS model.

Milky Way: As mentioned in Sect. 2.6, the RASS reveals bipolar hypershells, which make up the North Polar Spur and its counter-spurs. The observations are well fitted by the BHS model. A detailed modeling for the case of the Milky Way is described in Sofue (2000).

\section{Discussion}

\subsection{Probing starburst and halo gas distribution}

In the present shock approximation, the expansion velocity $v$ at radius $r$ is related to the mean ambient density $\rho_{0}$ and the explosion energy $E_{0}$ as $E_{0} \sim \rho_{0} r^{3} v^{2}$. Here, $r$ is measured from the observed shell radius, and the expansion velocity is also measured from the gaseous temperature inferred from X-ray spectrum. For given radii and velocities (temperature), which are both known parameters from observations, a scaling law can be established: in the case of constant $E_{0} / \rho_{0}$ values, we obtain identical shock envelopes.

For a given temperature (velocity), the emission measure is estimated from the intensity, and therefore, the accumulated total amount of gas in the shell is known, which is related to the mean gas density $\rho_{0}$. Hence, we can measure $\rho_{0}, r$ and $v$ from observations, and thus, we can finally estimate the total energy of the explosion.

The morphology of the BHS is sensitive to the density distribution in the disk and halo. The shell shape in the uppermost part manifests the halo-intergalactic density structure. If the intergalactic gas density is very low, the shell will have a larger on-average radius and a more conical form. In the case of higher density, the shell becomes compact and round. Among the various ambient density distributions in Table 1, Models S2 and S6 appear to better fit the observations than the other models. Although both models allow a sufficient global reproduction of the observed morphology of NGC 253, Model S6 appears to be preferable to reproduce the dumbbell shape of the observed X-ray lobes. This suggests that the disk-to-halo gas density is rather uniform until it faces the intergalactic low-density region. Such uniform density distribution may be possible if the gas is confined by the gas pressure, not hydrostatic equilibrium in the disk gravity. In order to clarify this and fit the observations in more detail, higher resolution imaging in the soft X-rays is necessary.

\subsection{Origin of the hypershells}

In our model, the energy injection at the galactic center is assumed to be impulsive, and the shock is strong enough to create a well-defined shell structure. The required total energy given to the interstellar gas is of the order of $\sim 10^{55}$ ergs. The time scale of the explosive event responsible for the energy release is assumed to be significantly shorter than the expansion timescale of the hypershell, $t \sim r / v \sim 10^{7}$ years for $r \sim$ several $\mathrm{kpc}$ and $v \sim$ $200 \mathrm{~km} \mathrm{~s}^{-1}$. So, the explosion (starburst) time scale must be shorter than a few million years. Such impulsiveness and robustness of the energy release can be explained if the nucleus of the galaxy has experienced a starburst $\sim 10^{7}$ years ago, lasting for a few million years or shorter, during which $\sim 10^{4}-10^{5}$ type II supernovae exploded. It is worth mentioning that similar timescales are predicted from starburst modeling based on infrared observations (e.g., Förster-Schreiber et al. 2000).

Alternative models have also been discussed by several authors: Suchkov et al. $(1994,1996)$ developed a massloading outflow model, and simulated the X-ray jets in M 82 using their hydrodynamical code. In their model, the blowout itself is as hot or as highly energetic as one would detect in the ROSAT band. This model may provide a better fit in the case of M 82 , where we observe a centrally-filled high energy flow well collimated in the direction perpendicular to the disk. A stellar-wind driven Galactic wind (Heckman et al. 1990) could also produce a shell structure, as observed with ROSAT for NGC 253 (Pietsch et al. 2000). If the duration of the starburst is short enough compared to the expansion time scale of the shell, namely, if the starburst is "impulsive" compared to the shell's life time, the wind model and the shocked BHS model give essentially the same result. On the other hand, if the wind is steady and longer-lived than the shell's expansion time, e.g. a few tens of million years, the flow would become an open-cone shape, which does not apply to round shells observed in the case of NGC 253.

\section{Summary and conclusions}

We presented a theoretical model of the million degrees phase of the interstellar medium in the bulge, disk and halo of spiral galaxies. In this model, we assume a sudden energy release in the nuclear region of the galaxy, as provided, e.g., by a nuclear starburst. The energy release is supposed to form a bipolar hyper-shell (BHS) of ionized ISM, which is heated to a million degrees and is thus visible in the soft X-ray band. Our simulations are based on the assumption of an adiabatic shock envelope, and our projections of the three-dimensional data cubes for different galaxy inclinations take into account the absorption of soft X-rays by intervening cold gas in the disk of the galaxy. The model predicts different spectral energy distributions of the soft X-ray emission for different distances from the galactic plane.

We applied our model to a mini-sample of nearby spiral galaxies, which are all classified as starburst galaxies, and the model-predictions are nicely supported by the observations. In the case of the very nearby galaxy NGC 253 $(d=2.6 \mathrm{Mpc})$, the X-ray images allow a high spatial resolution. Our model reproduces the ROSAT observations, namely the hourglass-shaped morphology, the absorption of disk and halo emission from the reverse (NW) side of the nearly edge-on galaxy due to cold gas in the disk, as well as the spectral distribution of the emission components. 
We extended our model to other spiral galaxies seen under different inclinations, edge-on (NGC 3079, M 82), under high inclination (NGC 4258, NGC 1808) or faceon (M 83). As for NGC 253, our simulations match the observed X-ray morphology and spectral energy distribution. The X-ray corona of the Milky Way, observed in the ROSAT All Sky Survey, can also be matched with our model. This topic is briefly discussed in this publication and is subject of another, more detailed publication (Sofue 2000).

From the comparison of our simulations with the observations, we are able to deduce expansion velocities of the shells between $200 \mathrm{~km} \mathrm{~s}^{-1}$ and $300 \mathrm{~km} \mathrm{~s}^{-1}$, which are necessary to heat the gas to $(2-4) 10^{6} \mathrm{~K}$. The age of the shells has to be of the order of some $10^{7}$ years, and from this results we can derive an upper limit for the duration and intensity of the initial starburst which provided the energy release to form the BHS morphology. In general, this energy release is estimated to be of the order of $10^{55} \mathrm{ergs}$ for all galaxies, and typical timescales of the burst seem to be $\lesssim$ several $10^{6}$ years. Such timescales are also predicted from infrared observations in the case of M 82, NGC 253 and NGC 1808 (e.g., Förster-Schreiber et al. 2000).

The new generation X-ray observatories, Chandra and $\mathrm{XMM}$, both now in orbit, have wider energy bands than ROSAT had $(0.2-10 \mathrm{keV}$ versus $0.1-2.4 \mathrm{keV})$ and offer superior spectral resolution. They allow us to study spiral galaxies at sub-arcsec resolution (Chandra) and to probe the ISM with an extremely high photon collecting power at a resolution of $\sim 10^{\prime \prime}$ (XMM). The combination of Chandra and XMM results will provide spatially highly resolved images of the bright ISM features, as well as detailed X-ray spectra of the ISM in general. The BHS model, in which the shell morphology is sensitive to the ambient density distribution, will then allow us to probe the distributions of the million degree interstellar medium in the disk, halo and intergalactic space as well as the disk halo interface at high spatial and spectral resolution.

Acknowledgements. We are grateful to Dr. W. Pietsch for helpful comments and providing the NGC 3079 and NGC 253 data. We would like to thank Sue Madden for carefully proofreading the manuscript.

\section{References}

Cecil, G., Morse, J. A., \& Veilleux, S. 1995, ApJ, 452, 316

Cox, P., \& Downes, D. 1996, ApJ, 473, 219
Dahlem, M., Hartner, G. D., \& Junkes, N. 1994, ApJ, 432, 598

Förster-Schreiber, N., et al. 2000, A\&A, in preparation

Haslam, C. G. T., Salter, C. J., Stoffel, H., \& Wilson, W. E. 1982, A\&AS, 47, 1

Heckman, T. M., Armus, L., \& Miley, G. K. 1990, ApJS, 74, 833

Hummel, E., Krause, M., \& Lesch, H. 1989, A\&A, 211, 266

Immler, S., Vogler, A., Ehle, M., \& Pietsch, W. 1999, A\&A, 352,415

Irwin, J. A., \& Seaquist, E. R. 1991, ApJ, 371, 110

Iwaki, H., Ueno, S., Koyama, K., Tsuru, T., \& Iwasawa, K. 1996, PASJ, 48, 409

Junkes, N., Zinnecker, H., Hensker, G., Dahlem, M., \& Pietsch, W. 1995, A\&A, 294, 8

Laumbach, D. D., \& Probstein, R. F. 1969, J. Fluid Mech., 35, 53

Lehnert, M. D., Heckman, T. M., \& Weaver, K. A. 1999, ApJ, 523, 575

Martin, P., Roy, J.-R., Noreau, L., \& Lo, K. Y. 1989, ApJ, 345,707

Möllenhoff, C. 1976, A\&A, 50, 105

Morrison, R., \& McCammon, D. 1983, ApJ, 270, 119

Nakai, N., Hayashi, M., Handa, T., Sofue, Y., Hasegawa, T., \& Sasaki, M. 1987, PASJ, 39, 685

Phillips, A. C. 1993, AJ, 105, 486

Pietsch, W., Trinchieri, G., \& Vogler, A. 1998, A\&A, 340, 351

Pietsch, W., Vogler, A., Klein, U., \& Zinnecker, H. 2000, A\&A, 360,24

Plante, R. L., Lo, K. Y., Roy, J.-R., Martin, P., \& Noreau, L. 1991, ApJ, 381, 110

Puche, D., \& Carignan, C. 1988, AJ, 95, 1025

Ryter, Ch. E. 1996, ApSpSc, 236, 285

Sakashita, S. 1971, ApSpSc, 14, 431

Snowden, S. L., Egger, R., Freyberg, M. J., et al. 1997, ApJ, 485,125

Sofue, Y. 1977, A\&A, 60, 327

Sofue, Y. 1984, PASJ, 36, 539

Sofue, Y. 1994, ApJL, 431, L91

Sofue, Y. 2000, ApJ, 540, 224

Strickland, D. K., Ponman, T. J., \& Stevens, I. R. 1997, A\&A, 320,378

Suchkov, A. A., Dinshaw, S. B., Heckman, T. M., \& Leitherer, C. 1994, ApJ, 430, 511

Suchkov, A. A., Berman, V. G., Heckman, T. M., \& Balsara, D. S. 1996, ApJ, 463, 528

Tomisaka, K., \& Ikeuchi, S. 1988, ApJ, 330, 695

Tomisaka, K., \& Bregman, J. N. 1993, PASJ, 45, 513

van Albada, G. D., \& van der Hulst, J. M. 1982, A\&A, 115, 263

van der Kruit, P. C. 1974, ApJ, 192, 1

Veron-Cetty, M. P., \& Veron, P. 1985, A\&A, 145, 425

Vogler, A., \& Pietsch, W. 1999a, A\&A, 342, 101

Vogler, A., \& Pietsch, W. 1999b, A\&A, 352, 64 